EPJ Web of Conferences 61, 01006 (2013)

DOI: 10.1051/epjconf/ 20136101006

(C) Owned by the authors, published by EDP Sciences, 2013

\title{
Polarimetric observations of the innermost regions of relativistic jets in X-ray binaries
}

\author{
D. M. Russell ${ }^{1,2, a}$ and T. Shahbaz ${ }^{1,2}$ \\ ${ }^{1}$ Instituto de Astrofísica de Canarias (IAC), E-38200 La Laguna, Tenerife, Spain \\ ${ }^{2}$ Departamento de Astrofísica, Universidad de La Laguna (ULL), E-38206 La Laguna, Tenerife, Spain
}

\begin{abstract}
Synchrotron emission from the relativistic jets launched close to black holes and neutron stars can be highly linearly polarized, depending on the configuration of the magnetic field. In X-ray binaries, optically thin synchrotron emission from the compact jets resides at infrared-optical wavelengths. The polarimetric signature of the jets is detected in the infrared and is highly variable in some X-ray binaries. This reveals the magnetic geometry in the compact jet, in a region close enough to the black hole that it is influenced by its strong gravity. In some cases the magnetic field is turbulent and variable near the jet base. In Cyg X-1, the origin of the $\gamma$-ray, $\mathrm{X}$-ray and some of the infrared polarization is likely the optically thin synchrotron power law from the inner regions of the jet. In order to reproduce the polarization properties, the magnetic field in this region must be highly ordered, in contrast to other sources.
\end{abstract}

\section{Introduction}

The polarization properties of emission from black hole X-ray binaries (BHXBs) have been well studied at radio frequencies [10], and have recently been given some attention at optical and infrared (IR) frequencies. In the optical regime, polarization due to the scattering of intrinsically unpolarized thermal emission can be modulated on the orbital period, which places constraints on the physical and geometrical properties of the system [8, 15]. At radio, and in some cases at optical/IR frequencies, variable polarization can be due to synchrotron emission from the jets launched via the process of accretion onto the black hole or neutron star in X-ray binaries [5, 16, 28, 29, 34].

Compact, conical jets are known to produce a flat or slightly inverted spectrum from radio to infrared frequencies in BHXBs $\left(0 \leq \alpha_{\text {thick }} \leq+0.5\right.$, where $F_{v} \propto v^{\alpha}$; $[11,14,27,30])$. Linear polarization has been detected from this optically thick flat spectrum at a level of a few per cent at radio frequencies in a few BHXBs (e.g. $[6,12])$. At some frequency, generally considered to lie in the infrared regime, this synchrotron spectrum breaks to one which is optically thin, with $-1 \leq \alpha_{\text {thin }} \leq-0.4$. The power law spectrum of the optically thin emission has been identified and isolated in several BHXBs in the infrared/optical in the hard state $[17,19,30]$ (we adopt the state definitions of ref. [1]). Spectral evidence for synchrotron emission from jets in quiescent BHXBs also exists [13, 35].

\section{Polarization of compact jets}

To date, few studies have attempted to uncover the polarimetric signature of the optically thin synchrotron emission from compact jets. This emission originates close to the base of the jet, in a region likely associated with the start of the particle acceleration in the jet (e.g. [25]). Optically thin synchrotron emission is intrinsically polarized. If the local magnetic field in the emitting region is uniform (ordered), a net linear polarization is observed. If the field is tangled, the differing angles of the polarized light suppress the observed, average polarization. The maximum polarization strength is $\sim 70-80$ per cent, in the case of a perfectly ordered field [3] and is dependent only on the degree of ordering of the field and the energy distribution of the electron population. Polarimetric measurements of the optically thin power law therefore provide a powerful tool to uncover the nature of the magnetic field structure in this region, which is important for models and simulations of jet production.

In the optical/IR regime of X-ray binaries, other components such as the accretion disc and companion star often dominate, suppressing any polarization from synchrotron, but when the synchrotron makes a strong contribution, intrinsic polarization has been detected [e.g. $7,9,28,29,34]$. Scattering and interstellar absorption cannot reproduce the observed wavelength dependence of the fractional linear polarization (FLP) in Sco X-1, Cyg X-2 and GRO J1655-40. The FLP is on the order of $~ 1-10$ per cent. The PA is usually approximately orthogonal to the axis of the resolved radio jet when this angle is known, which implies the magnetic field is parallel to the jet axis. For a highly ordered magnetic field, the FLP from opti-

\footnotetext{
a. e-mail: russell@iac.es
}

This is an Open Access article distributed under the terms of the Creative Commons Attribution License 2.0, which permits unrestricted use, distribution, and reproduction in any medium, provided the original work is properly cited. 
cally thin synchrotron emission could be as high as $\sim 70 \%$ [3]. The levels detected (a few per cent at most) suggest a tangled magnetic field is present.

\subsection{Evidence for variability}

The IR polarization of FLP $\sim 6$ per cent in GRO J1655-40 was measured during outburst when the source was in the hard state [28], whereas FLP $\leq 1.3 \%$ was measured at the same wavelength during quiescence [9], so the outburst polarization must have been transient. Short timescale variability was discovered in the IR polarization of Sco X-1 [28]. Follow-up fast-timing optical polarimetry of Sco X-1 revealed short flares of enhanced FLP with position angles similar to the IR polarization [29]. The implication is that the optical emission from Sco $\mathrm{X}-1$ is sometimes polarized by several per cent, but on timescales of 10 seconds or less. The flares were present during an $\mathrm{X}$-ray state in which radio jets are known to be produced. The flares of polarization could arise due to brief ordering of the predominantly tangled magnetic field near the jet base, or intermittency of the jets themselves.

In addition, the near-infrared (NIR) FLP of GX 3394 was found to be variable, and a correlation between the Stokes q and u polarization vectors was discovered [29]. These data were taken during the hard state, and the flux spectrum is consistent with the jet dominating the NIR emission at the time. The polarization signature of the jet appears to be changing rapidly. The NIR and optical observations of X-ray binaries to date are consistent with a variable, predominantly tangled magnetic field geometry in the jet, with field lines preferentially orientated along the jet axis.

\section{The multi-wavelength polarization of Cygnus X-1}

More than thirty years ago, the linear polarization of Cyg X-1 was measured to be $2-5$ per cent at $2.5-5.2 \mathrm{keV}$ [21]. More recently, the $\gamma$-ray polarization of Cyg X-1 was found to be very high, $67 \pm 30$ per cent at $0.4-2 \mathrm{MeV}$ using the IBIS instrument on board INTEGRAL [20], later confirmed using the SPI instrument, $76 \pm 15$ per cent at $0.23-0.85 \mathrm{MeV}$ [18]. The only viable mechanism for producing such high polarization at these energies is optically thin synchrotron emission, and it was claimed that the high energy electrons in the jet are the origin of the polarization $[18,20]$. Detailed spectral modelling of the keV-MeV and $\mathrm{keV}-\mathrm{GeV}$ (e.g. [22, 23, 38]) emission of Cyg X-1 has confirmed the presence of an $\mathrm{MeV}$ tail in the hard state, which could either be due to hybrid Comptonization or a synchrotron component. If the high levels of polarization at $0.2-2 \mathrm{MeV}$ are robust, synchrotron is the favoured mechanism, and jet models are consistent with the MeV tail being the high energy extension of the optically thin synchrotron power law extending from infrared wavelengths [22, 27, 37, 38]. In [31] we present new, high-precision NIR polarization measurements of Cyg X-1. We gather archival flux spectral energy distributions (SEDs) and all polarization measurements of the source published to date, and attempt to model the multi-wavelength flux spectrum, FLP spectrum and PA spectrum self-consistently. The main results are presented in figure 1.

The broadband, radio to $\gamma$-ray flux density $\left(F_{v}\right)$ spectrum of $\mathrm{Cyg} X-1$ in the hard state is presented in the upper left panel of figure 1. Here, we adopt a simple, toy model of a synchrotron jet, a blackbody from the companion star, and a Comptonized corona approximated by a power law with an exponential cutoff. The aim is to approximately reproduce the observed spectrum phenomenologically, in order to use this as an input spectrum for the polarization model. The supergiant $\mathrm{O}$ star dominates the IR/optical/UV emission and can be approximated by a single temperature blackbody. The jet consists of a broken power law describing the optically thick and optically thin regions of the synchrotron spectrum, with some curvature at the frequency of the spectral break, $v_{\mathrm{b}}$. The total spectrum is the sum of the synchrotron, blackbody and Comptonized corona. We compare the observed broadband polarization properties of Cyg X-1 with that expected from a synchrotron jet, the spectrum of which (and its contribution to the total flux) is defined here by the model approximating the flux spectrum. We see from figure 1 that as expected, the jet dominates the radio-mm-mid-IR spectrum, the companion becomes the brightest emitter in the mid-IR-NIR-opticalUV regime, the corona dominates the X-ray flux and the jet spectrum accounts for the $\gamma$-ray $\mathrm{MeV}$ tail (at $v \sim 10^{20}$ $\mathrm{Hz}$; see also [18, 20, 27]). It has been demonstrated that synchrotron self-Compton emission (SSC) and/or Compton upscattering of blackbody stellar emission can account for the $\mathrm{GeV}\left(v \sim 10^{22}-10^{24} \mathrm{~Hz}\right)$ flux in the hard state $[22,38]$. Since no $\mathrm{GeV}$ polarization measurements have been made, and the GeV-emitting component seems not to dominate at energies lower than the $\mathrm{GeV}$ regime, we have no need to include an extra component in our model at $\mathrm{GeV}$ energies.

The expected linear polarization of optically thin synchrotron emission is (see [3]), $F L P_{\text {thin }}=f \frac{p+1}{p+7 / 3}=$ $f \frac{1-\alpha_{\text {thin }}}{5 / 3-\alpha_{\text {thin }}}$, where $f$ represents the ordering of the magnetic field and $p$ is the electron energy distribution. For optically thin synchrotron emission with a typical spectral index of $\alpha_{\text {thin }} \sim-0.7(p=2.4)$, the maximum polarization (for an ordered magnetic field, i.e. $f=1$ ) is therefore $F L P_{\text {thin }}=72$ per cent. Optically thick (absorbed) synchrotron radiation is expected to be less polarized than optically thin synchrotron (e.g. [4]), $F L P_{\text {thick }}=f \frac{3}{6 p+13}$, with a position angle that differs by $90^{\circ}$ to that of optically thin synchrotron polarization. The maximum polarization from this spectrum is $F L P_{\text {thick }}=11$ per cent for $p=2.4$. If the magnetic field ordering and PA remain constant down the length of the jet, then this equation can describe the polarization expected from a flat/inverted optically thick jet spectrum composed of overlapping synchrotron spectra. If however, the ordering changes or the PA varies with distance from the jet base (e.g. in helical fields), the polarization will change as a function of frequency in the optically thick spectrum. 

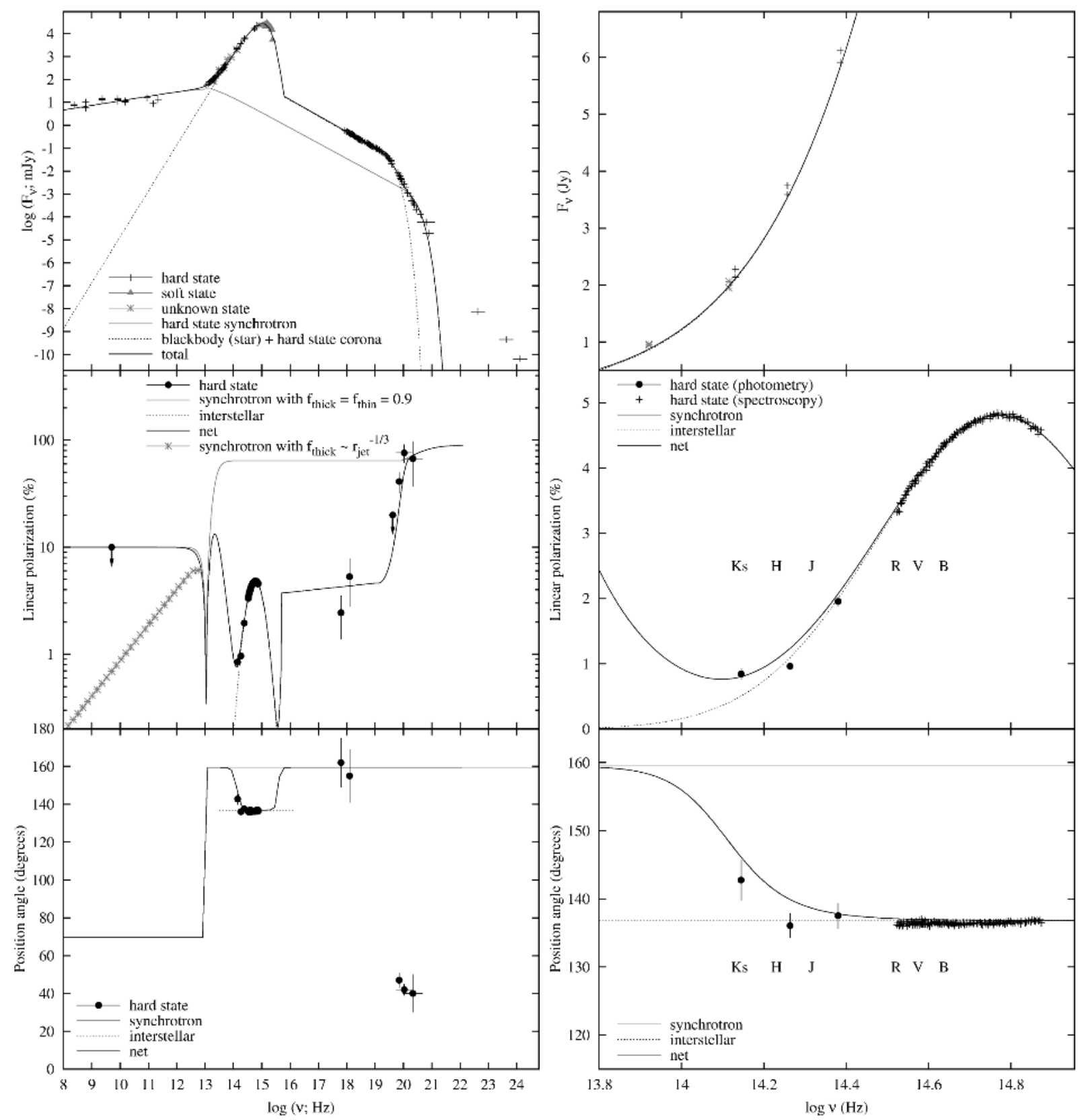

Figure 1. Left: Radio to $\gamma$-ray flux density spectrum ( $F_{v}$; upper panels), FLP spectrum (centre panels) and polarization PA (lower panels) of Cyg X-1. For data references and details of the model, see Russell \& Shahbaz (2013; [31]). Right: The same as the left panels but just the IR-optical region, showing how the interstellar model approximates the IR-optical data (see also [24]), except in $K_{\mathrm{S}}$-band.

The above equations are used to predict the FLP of the jet spectrum. For the optically thick spectrum, we consider the case of a constant ordering and PA of the magnetic field along the jet, and also a jet in which the field ordering changes with distance along the jet. At IR/optical/UV frequencies the companion star dominates which, by definition for a blackbody, is expected to be unpolarized. Optical FLP values of 3-5 per cent are well documented in the literature for Cyg X-1, and almost all of this FLP has an interstellar dust origin. A small fraction of optical FLP has been found to be due to scattering in the stellar wind of the companion and varies on the orbital period [24]. We adopt the interstellar dust polarization model of [33], which has been fitted to optical spectra of Cyg X-1 by [24]. The detail of the model and data in the optical-IR region can be seen in right panels of figure 1. Photons from the Comptonized corona may be emitted isotropically and if no relativistic, beaming or bulk motion effects are present then one may expect a very low net polarization for the corona. In the commonly used Comptonized corona model the seed photons are from the disc (but some may also be from the jet), so the corona is expected to be not much more than 5 per cent polarized (see also [26, 32]). In our toy model we assume zero polarization for this component. 
The resulting FLP spectrum and PA spectrum are shown in the centre and lower panels of figure 1, respectively (see [31] for model details). We adopt the values $f=0.9$ and $P A_{\text {thin }}=159.5^{\circ}$ (which is the mean position angle of the radio jet on the plane of the sky; [36]). By choosing these values the observed FLP and PA at all frequencies can be recovered by the model, with one exception: the PA of the $\gamma$-ray polarization differs to that of the model by $\sim 60^{\circ}$, which appears to imply a field that is mis-aligned with the jet axis in the $\gamma$-ray emitting region of the jet. The high value of $f$ is required in order to produce a synchrotron spectrum that can account for the very high $\gamma$-ray FLP and the lower FLP in X-ray. In the case of a constant value of $f$ along the length of the jet, the model predicts that the FLP in radio is $\sim 10$ per cent, which is just consistent with the observed upper limit [36]. We find that the NIR $J$ and $H$-band data fit very well on the extrapolation of the interstellar dust model, but the $K_{\mathrm{S}}$-band has a slightly higher FLP (see figure 1, right panels). The $K_{\mathrm{S}}$-band data are consistent with the model whereby the highly polarized jet synchrotron emission makes a small contribution. The observed $K_{\mathrm{S}}$-band PA is $\sim 6^{\circ}$ higher than the $J$ and $H$-bands, and consistent with the smooth transition expected from the jet model.

In our simple toy model the PA is constant for optically thin synchrotron emission [3, 4]. However, electron distributions with a sharp break or cut-off could produce synchrotron emission with frequency-dependent PA [2]. Numerical solutions have shown that the rate of change of PA with frequency can be large, especially for steeper spectra, while FLP may increase by only a factor $\leq 2$ [2]. Since these are numerical and not analytical solutions, we have not included them in our model. Nevertheless, the curved $\gamma$-ray spectrum leads to the possibility that a change of PA could be expected. In the numerical results of [2], the PA changes smoothly by up to $\sim 60^{\circ}$ over two orders of magnitude in frequency. This may therefore explain the apparent shift of $60^{\circ}$ between the X-ray PA and the $\gamma$-ray PA, both of which originate in the optically thin synchrotron emission from the jet in our model.

\section{Conclusions}

The magnetic field near the base of the jet of Cyg X-1 appears to be highly ordered, orthogonal to the jet axis and stable over several years. This is astonishing since in other $\mathrm{X}$-ray binaries and AGN the magnetic field ordering in this region of the jet is usually found to be much lower. In BHXBs and some neutron star X-ray binaries, the FLP is a few per cent and the magnetic field lines are usually preferentially parallel to the jet axis, with some evidence for variability on short timescales, even in cases where synchrotron emission appears to dominate the flux (see [29] and references therein). However, only a few systems have been studied to date. The highly ordered magnetic field in the jet of Cyg X-1 is unprecedented, however Cyg X-1 is the first HMXB in which this has been well measured, and could imply a different field geometry in this type of accreting system.

\section{References}

[1] Belloni T. M., in The Jet Paradigm - From Microquasars to Quasars (Lect. Notes Phys., Springer, Berlin Heidelberg, 2010), 794

[2] Björnsson C.-I., MNRAS, 216, 241 (1985)

[3] Björnsson C.-I., Blumenthal G. R., ApJ, 259, 805 (1982)

[4] Blandford R., Agol, E., Broderick A., Heyl J., Koopmans L., Lee H.-W., in Proc. XII Canary Islands Winter School of Astrophysics, Astrophysical Spectropolarimetry (Cambridge Univ. Press, Cambridge, UK, 2002), 177

[5] Brocksopp C., Miller-Jones J. C. A., Fender R. P., Stappers B. W., MNRAS, 378, 1111 (2007)

[6] Brocksopp C., Corbel S., Tzioumis A., Broderick J. W., Rodriguez J., Yang J., Fender R. P., Paragi Z., MNRAS, 432, 931 (2013)

[7] Chaty S., Dubus G., Raichoor A., A\&A, 529, 3 (2011)

[8] Dolan J. F., Tapia S., PASP, 101, 1135 (1989)

[9] Dubus G., Chaty S., A\&A, 458, 591 (2006)

[10] Fender R. P., in Compact Stellar X-Ray Sources (Cambridge University Press, Cambridge, UK, 2006), 381

[11] Fender R. P., Pooley G. G., Durouchoux P., Tilanus R. P. J., Brocksopp C., MNRAS, 312, 853 (2000)

[12] Gallo E., Corbel S., Fender R. P., Maccarone T. J., Tzioumis A. K., MNRAS, 347, L52 (2004)

[13] Gallo E., Migliari S., Markoff S., Tomsick J. A., Bailyn C. D., Berta S., Fender R., Miller-Jones J. C. A., ApJ, 670, 600 (2007)

[14] Gandhi P., et al., ApJ, 740, L13 (2011)

[15] Gliozzi M., Bodo G., Ghisellini G., Scaltriti F., Trussoni E., A\&A, 337, L39 (1998)

[16] Hannikainen D. C., Hunstead R. W., CampbellWilson D., Wu K., McKay D. J., Smits D. P., Sault R. J., ApJ, 540, 521 (2000)

[17] Hynes R. I., et al., MNRAS, 345, 292 (2003)

[18] Jourdain E., Roques J. P., Chauvin M., Clark D. J., ApJ, 761, 27 (2012)

[19] Kalemci E., et al., ApJ, 622, 508 (2005)

[20] Laurent P., Rodriguez J., Wilms J., Cadolle Bel M., Pottschmidt K., Grinberg V., Science, 332, 438 (2011)

[21] Long K. S., Chanan G. A., Novick R., ApJ, 238, 710 (1980)

[22] Malyshev D., Zdziarski A. A., Chernyakova M., MNRAS, 434, 2380 (2013)

[23] McConnell M. L., et al., ApJ, 572, 984 (2002)

[24] Nagae O., Kawabata K. S., Fukazawa Y., Okazaki A., Isogai M., Yamashita T., AJ, 137, 3509 (2009)

[25] Polko P., Meier D. L., Markoff S., ApJ, 723, 1343 (2010)

[26] Poutanen J., ApJS, 92, 607 (1994)

[27] Rahoui F., Lee J. C., Heinz S., Hines D. C., Pottschmidt K., Wilms J., Grinberg V., ApJ, 736, 63 (2011)

[28] Russell D. M., Fender R. P., MNRAS, 387, 713 (2008) 
[29] Russell D. M., Casella P., Fender R., Soleri P., Pretorius M. L., Lewis F., van der Klis M., in Proceedings of 'High Time Resolution Astrophysics IV - The Era of Extremely Large Telescopes - HTRA-IV' (Proceedings of Science, Agios Nikolaos, Crete, Greece, 2011) (arXiv:1104.0837)

[30] Russell D. M., et al., 2013, ApJ, 768, L35 (2013)

[31] Russell D. M., Shahbaz T., 2013, MNRAS, submitted

[32] Schnittman J. D., Krolik J. H., ApJ, 712, 908 (2010)

[33] Serkowski K., Mathewson D. S., Ford V. L., ApJ, 196, 261 (1975)
[34] Shahbaz T., Fender R. P., Watson C. A., O’Brien K., ApJ, 672, 510 (2008)

[35] Shahbaz T., Russell D. M., Zurita C., Casares J., Corral-Santana J. M., Dhillon V. S., Marsh T. R., MNRAS, 434, 2696 (2013)

[36] Stirling A. M., Spencer R. E., de la Force C. J., Garrett M. A., Fender R. P., Ogley R. N., MNRAS, 327, 1273 (2001)

[37] Zdziarski A. A., Lubiński P., Sikora M., MNRAS, 423, 663 (2012)

[38] Zdziarski A. A., Pjanka P., Sikora M., 2013, MNRAS, submitted (arXiv:1307.1309) 